\title{
Overcoming the transcatheter aortic valve replacement Achilles heel: conduction abnormalities - a systematic review
}

\author{
Alberto Alperi, Guillem Muntané-Carol, Afonso B. Freitas-Ferraz, Lucia Junquera, David del Val, \\ Laurent Faroux, François Philippon, Josep Rodés-Cabau \\ Quebec Heart \& Lung Institute, Laval University, Quebec City, Quebec, Canada \\ Correspondence to: Josep Rodés-Cabau, MD. Quebec Heart \& Lung Institute, Laval University, 2725 Chemin Ste-Foy, G1V4G5, Quebec City, \\ Quebec, Canada. Email: josep.rodes@criucpq.ulaval.ca.
}

\begin{abstract}
Background: Transcatheter aortic valve replacement (TAVR) has been shown to be a good alternative to surgery for treating severe symptomatic aortic stenosis (AS) across the whole range of surgical risk patients. Whereas most periprocedural TAVR complications have significantly decreased over time, conduction disturbances remain high. Approaches to decrease this shortcoming are under continuous investigation.

Methods: We conducted a systematic review focusing on modifiable factors impacting post-TAVR conduction disturbances, such as balloon aortic valvuloplasty (BAV), type of new-generation transcatheter valve and implantation depth (ID). Search strategies were based on the best available evidence from each study. Primary endpoints were post-TAVR need of permanent pacemaker implantation (PPI) and new onset left bundle branch block (NOLBBB).

Results: Data from 35 studies with a total of 29,982 patients were analyzed. BAV did not negatively impact PPI rates after TAVR. In propensity-matched and randomized trials, the Evolut $\mathrm{R}$ valve was associated with higher rates of PPI compared to the Sapien 3 valve (25\% vs. $19.2 \%$ in propensity-matched studies; $22.9 \%$ vs. $19 \%$ in a randomized trial). The Acurate Neo valve was associated with the lowest PPI rate in observational studies $(10.4 \%)$, but a PPI rate similar to Sapien 3 was reported in a randomized trial (10\% vs. 9\%). The Portico valve system was associated with a higher PPI risk (PPI rate of $21.9 \%$ and $27.7 \%$ in propensitymatched and randomized studies, respectively). ID and its relation with the membranous septum (MS) length predicted post-TAVR conduction disturbances, particularly with Evolut R and Sapien 3 valves.

Conclusions: Pre-TAVR BAV did not increase the risk of conduction disturbances post-TAVR. Among the new-generation transcatheter valve systems, Sapien 3 and Acurate Neo valves were associated with the lowest PPI rates followed by the Evolut and Portico valves. A deeper valve implantation and a shorter MS length determined an increased risk of conduction disturbances post-TAVR.
\end{abstract}

Keywords: Transcatheter aortic valve replacement (TAVR); pacemaker; left bundle branch block; conduction disturbances

Submitted Mar 09, 2020. Accepted for publication Apr 07, 2020.

doi: $10.21037 /$ acs-2020-av-40

View this article at: http://dx.doi.org/10.21037/acs-2020-av-40

\section{Introduction}

Transcatheter aortic valve replacement (TAVR) is a wellestablished therapy for treating patients with severe symptomatic aortic stenosis (AS). Most recent clinical guidelines on valvular heart disease management have broadened its indications according to the results of several randomized clinical trials (1-4). Moreover, recent data on low surgical risk patients have shown TAVR outcomes to be equivalent or superior to standard surgical aortic valve replacement (SAVR) $(5,6)$. However, TAVR still faces important shortcomings, and procedural-related conduction disturbances are currently considered its main Achilles heel. Permanent pacemaker implantation (PPI) as well as new onset left bundle branch block (NOLBBB) after TAVR have 
been shown to be independent predictors of all-cause death and heart failure hospitalization (7). PPI incidence has been high in TAVR registries $(\sim 15 \%)$, with the transcatheter approach exhibiting higher PPI rates than SAVR in most observational and randomized studies (3-8).

The aim of this systematic review was to offer the most up-to-date evidence on new-onset bradyarrhythmia and conduction disturbances with new-generation TAVR devices, focusing on modifiable procedural factors that may play a role on the aforementioned outcomes.

\section{Methods}

\section{Search strategy}

A systematic review of available data reporting outcomes on conduction disturbances post-TAVR was performed in accordance with the guidance and the reporting items specified on the Preferred Reported Items for Systematic Reviews and Meta-Analysis (PRISMA) statement (9). Three main subjects were considered: pre-TAVR balloon aortic valvuloplasty (BAV), type of valve selection, and prosthesis implantation depth (ID). A computerized search was performed on PubMed and Embase databases in order to identify any relevant entry, as well as manual search of primary studies references (backward snowballing). TAVR in patients with previous aortic bioprostheses (valve-in-valve procedures), as well as TAVR indications beyond severe AS, were out of the scope of this review. We limited our search to studies including new-generation TAVR devices: Acurate neo (Boston Scientific, Natick, MA, USA); Evolut R or Evolut PRO (Medtronic, Minneapolis, MN, USA); Portico (St. Jude Medical, Saint Paul, MN, USA) and Sapien 3 (Edwards Lifesciences, Irvine, CA, USA). Databases were last accessed January 31st 2020, and studies were included if they were published in English. Data were extracted using a standardized data abstraction sheet. Two investigators (AA and GM) conducted the literature search, selection and data extraction in duplicate. Any discrepancies between these two investigators were resolved by a third investigator (JRC). Clinical characteristics, as well as in-hospital and/or 30-day outcomes on new PPI or NOLBBB were collected as reported by authors. The Newcastle-Ottawa scale was used for quality assessment of non-randomized studies selected (Table S1).

The search strategy was conducted differently according to the variable robustness of evidence available to address each research question.

\section{Balloon aortic valvuloplasty prior to TAVR}

Only observational propensity-matched studies and randomized clinical trials using new-generation devices were included. For studies including both early and newgeneration valves, only those in which the latter represented more than $80 \%$ of the whole cohort, or if a separate analysis for new-generation valves was carried out, were selected. The following key terms were used: transcatheter aortic valve replacement/implantation valvuloplasty; transcatheter aortic valve replacement/implantation predilation. A flow diagram illustrating BAV selection process is available in Figure S1.

\section{Direct valve type comparison}

Only observational propensity-matched studies and randomized clinical trials using new-generation devices were included. Studies including both early and new-generation valves were selected if either the latter represented more than $80 \%$ of the whole cohort, or if a separate endpoint analysis for new-generation valves was carried out. The following key terms were used: transcatheter aortic valve replacement/implantation comparison; transcatheter aortic valve replacement/implantation selection; Sapien 3 Evolut; Sapien 3 Acurate Neo; Evolut Acurate Neo; Sapien 3 Portico; Evolut Portico.

\section{Implantation depth}

Observational studies reporting any association (whether positive, negative or null) between ID and post-TAVR conduction abnormalities. Studies should include at least 100 new-generation valve recipients. The following key terms were used: transcatheter aortic valve replacement/ implantation pacemaker; transcatheter aortic valve replacement/implantation predictors. After title and abstract revision, as well as duplicity elimination, the following terms were used: "depth", "height" and "pacemaker".

\section{Endpoints}

Primary outcomes of the systematic review were short-term new PPI and NOLBBB, whether at hospital discharge or at 30-day follow-up, as reported by the authors.

\section{Statistical analysis}

Continuous variables were expressed as mean \pm SD. Global cohort values were reported as weighted mean (95\% confidence interval) or frequency (percentage). Weighted 
mean was calculated according to the total number of patients in each study (weight $=n$ ). Data originated from propensity-matched investigations were analyzed separately from the evidence derived from clinical trials. Data analyses were performed using the STATA software (v14.0; StataCorp).

\section{Results}

\section{Balloon aortic valvuloplasty prior to TAVR}

PubMed and Embase searches identified 176 and 353 records, respectively. After title and abstract revision, as well as duplicity elimination, 5 studies fulfilled the criteria and were selected (10-14). Clinical characteristics and outcomes were collected as reported by the authors and are summarized in Table 1. Quantitative data from Spaziano et al. (11) were reported as weighted means since the BAV group was subdivided in selective and systematic predilation.

Overall, 2,412 patients were evaluated, 1,231 undergoing direct TAVR and 1,181 with a pre-BAV approach. Clinical characteristics and conduction disturbances are depicted in Table 1. Most patients received either a Sapien $3(84.9 \%)$ or Evolut R (11.6\%) valve. Only 2.6\% of the cohort received an early-generation valve such as CoreValve (Medtronic, Minneapolis, MN, USA) or Lotus (Boston Scientific, Natick, MA, USA).

Rates of new PPI after TAVR were similar between those undergoing BAV versus patients with direct valve implantation. No information regarding NOLBBB was reported in these studies. The only randomized data, reported by Toutouzas et al. (14). showed no differences between the two strategies in self-expandable prosthesis recipients (30-day new PPI $32.8 \%$ in direct TAVR $v s$. $27.5 \%$ in BAV pre-TAVR, $\mathrm{P}=0.54)$. Studies focusing exclusively on balloon-expandable devices reported either non-significant differences, like Abramowitz et al. (10) (odds ratio 1.11, 95\% CI: 0.44-2.80), or even a higher rate of PPI in patients undergoing the direct TAVR approach (13.9\% vs. $10.4 \%, \mathrm{P}=0.032$ ) (13). Spaziano et al. (11) reported a lack of statistically significant differences after a separate evaluation of systematic predilation $v s$. direct TAVR (30-day new PPI: $4 \%$ vs. $27 \%$, respectively, $\mathrm{P}=0.16$ ) and selective predilation $v s$. direct TAVR (30-day new PPI: $18 \%$ vs. $18 \%$ direct TAVR, $\mathrm{P}=0.94$ ).

Global results showed similar rates of 30-day new PPI between both strategies: $16 \%$ with direct-TAVR and 13.2\% in the previous BAV group.

\section{Direct valve type comparison}

PubMed and Embase searches identified 606 and 2,374 records, respectively. After title and abstract revision, as well as duplicity elimination, 12 studies fulfilled the pre-specified criteria and were finally selected.

\section{Sapien 3 vs. Evolut R/PRO}

Data from 5 different studies (4 observational propensitymatched and 1 randomized trial) were included. Propensitymatched studies are summarized in Table 2. Overall, 23,965 patients were evaluated, 12,006 and 11,959 Sapien 3 and Evolut R/PRO recipients, respectively. Rates of new PPI across propensity-matched studies were slightly higher when using the Evolut R/PRO valve in comparison with the Sapien 3 valve (23.8\% Evolut vs. $18.4 \%$ Sapien 3). Three out of 5 studies reported significant differences regarding 30-day or post-procedural new PPI, all of them favoring the Sapien 3 valve $(15,17,18)$. Contrarily, the only randomized trial (19) did not find any differences in PPI rates between the two valves (22.9\% Evolut R vs. 19\% Sapien 3, $\mathrm{P}=0.34$ ) (Figure 1). The only study reporting data on NOLBBB after TAVR showed no significant differences between valve types after propensity matching (26.7\% Evolut R vs. 24.8\% Sapien 3, P=0.75) (16).

\section{Sapien 3 vs. Acurate Neo}

Data from 5 different studies (4 observational propensitymatched and 1 randomized trial) were included. The main characteristics of propensity-matched studies are summarized in Table 3. A total of 2,194 patients were evaluated, 1,250 Sapien 3 and 944 Acurate Neo recipients. Overall incidence of new PPI was 15.6\% for Sapien 3 and $10.4 \%$ for Acurate Neo across the propensity-matched studies. No data was available on NOLBBB.

One of the observational studies included exclusively patients with previous right bundle branch block (RBBB) and without previous PPI (21). Two of the observational studies reported higher PPI rates with Sapien 3: 15.5\% vs. 9.9\% Acurate $\mathrm{Neo}, \mathrm{P}=0.02$ (all-comers) (20); and 43.9\% Sapien 3 vs. $29.6 \%$ Acurate Neo, $\mathrm{P}=0.02$ (RBBB patients) (21); whereas the other matched studies reported numerically higher rates but not-statistically significant differences: $15.2 \%$ Sapien 3 vs. $12 \%$ Acurate Neo, $\mathrm{P}=0.068$ (24); and $16.4 \%$ Sapien 3 vs. 10.6\% Acurate Neo, $\mathrm{P}=0.24$ (23). Conversely, a recently published randomized trial showed no differences in PPI rates between valves (9\% Sapien 3 vs. 10\% Acurate Neo, $\mathrm{P}=0.76$ ), mainly driven by a much lower incidence of new PPI after Sapien 3 


\begin{tabular}{|c|c|c|c|c|c|c|c|c|c|}
\hline Study & Cohorts & $\begin{array}{l}\text { Patients } \\
\text { (N) }\end{array}$ & Age & STS & $\begin{array}{l}\text { Previous } \\
\text { PM }\end{array}$ & $\begin{array}{l}\text { Previous } \\
\text { RBBB }\end{array}$ & $\begin{array}{l}\text { Post-TAVR } \\
\text { PPI }\end{array}$ & $\begin{array}{l}\text { Type of } \\
\text { study }\end{array}$ & Devices \\
\hline \multirow[t]{2}{*}{$\begin{array}{l}\text { Abramowitz } \\
\text { et al. (10) }\end{array}$} & Direct & 119 & $82 \pm 7.9$ & $6.4 \pm 3.3$ & 15 (12.6) & NR & $12(11.5)$ & $\begin{array}{l}\text { Propensity- } \\
\text { matched }\end{array}$ & Sapien 3 \\
\hline & BAV & 126 & $82.1 \pm 7.8$ & $6.3 \pm 2.7$ & $22(17.5)$ & NR & $18(17)$ & & \\
\hline \multirow[t]{2}{*}{$\begin{array}{l}\text { Spaziano } \\
\text { et al. (11) }\end{array}$} & Direct & 116 & $\begin{array}{l}* 83.5 \\
(83.1-83.8)\end{array}$ & $\begin{array}{l}{ }^{*} 5.2 \\
(5-5.4)\end{array}$ & 15 (12.9) & $\begin{array}{l}15 / 91 \\
(16.5)\end{array}$ & 19 (20.9) & $\begin{array}{l}\text { Propensity- } \\
\text { matched }\end{array}$ & Sapien 3 \\
\hline & BAV & 58 & $\begin{array}{l}* 84 \\
(83.7-84.3)\end{array}$ & $\begin{array}{l}{ }^{*} 5.8 \\
(5.7-5.9)\end{array}$ & $5(8.6)$ & $\begin{array}{l}6 / 47 \\
(12.8)\end{array}$ & $6(11.3)$ & & \\
\hline \multirow[t]{2}{*}{$\begin{array}{l}\text { Giordano } \\
\text { et al. (12) }\end{array}$} & Direct & 139 & $82.6 \pm 5.8$ & $7.9 \pm 7.9$ & $13(9.4)$ & NR & 15 (10.8) & $\begin{array}{l}\text { Propensity- } \\
\text { matched }\end{array}$ & $\begin{array}{l}\text { Acurate Neo (5.1\%); CoreValve } \\
\text { (10\%); Evolut R (45.9\%); } \\
\text { Sapien } 3(30.2 \%) \text {; Portico (3.7\%); } \\
\text { Lotus }(5.1 \%)\end{array}$ \\
\hline & BAV & 139 & $83.1 \pm 6.3$ & $11.5 \pm 11.9$ & 27 (19.5) & NR & $12(8.6)$ & & \\
\hline \multirow[t]{2}{*}{$\begin{array}{l}\text { Dumonteil } \\
\text { et al. (13) }\end{array}$} & Direct & 772 & $81.7 \pm 6.7$ & $6 \pm 5.9$ & $88(11.4)$ & 77 (18.4) & 107 (13.9) & $\begin{array}{l}\text { Propensity- } \\
\text { matched }\end{array}$ & Sapien 3 \\
\hline & BAV & 772 & $81.6 \pm 6.6$ & $7.8 \pm 8.3$ & 89 (11.5) & 70 (18.4) & $80(10.4)$ & & \\
\hline \multirow[t]{2}{*}{$\begin{array}{l}\text { Toutouzas } \\
\text { et al. (14) }\end{array}$} & Direct & 85 & $81.3 \pm 6.9$ & NR & $18(21.2)$ & NR & $22(32.8)$ & $\begin{array}{l}\text { Randomized } \\
\text { trial }\end{array}$ & $\begin{array}{l}\text { Evolut R } 83.6 \% \text {; Evolut PRO } \\
4.7 \% \text {; CoreValve } 11.7 \%\end{array}$ \\
\hline & BAV & 86 & $82.1 \pm 7.4$ & NR & $17(19.8)$ & NR & $19(27.5)$ & & \\
\hline \multirow[t]{2}{*}{ Overall $^{\star}$} & Direct & 1,231 & $\begin{array}{l}82 \\
(81.9-82.1)\end{array}$ & $\begin{array}{l}6.51 \\
(6.4-6.6)\end{array}$ & $\begin{array}{l}149 / 1,231 \\
(12.1)\end{array}$ & $\begin{array}{l}92 / 863 \\
(10.7)\end{array}$ & $\begin{array}{l}175 / 1,094 \\
(16.0)\end{array}$ & - & $\begin{array}{l}\text { Sapien 3: 2,047/2,412 (84.9\%); } \\
\text { Evolut R/PRO: 279/2,412 (11.6\%); } \\
\text { Acurate Neo: 13/2,412 (0.5\%); } \\
\text { Portico: } 10 / 2,412(0.4 \%) ; \\
\text { CoreValve: 50/2,412 (2.1\%); } \\
\text { Lotus: } 13 / 2,412(0.5 \%)\end{array}$ \\
\hline & BAV & 1,181 & $\begin{array}{l}82.2 \\
(82.1-82.3)\end{array}$ & $\begin{array}{l}7.61 \\
(7.58-7.62)\end{array}$ & $\begin{array}{l}160 / 1,181 \\
(13.6)\end{array}$ & $\begin{array}{l}76 / 819 \\
(9.3)\end{array}$ & $\begin{array}{l}135 / 1,023 \\
(13.2)\end{array}$ & & \\
\hline
\end{tabular}

Values are mean $\pm \mathrm{SD}$ or $\mathrm{n}(\%)$ except as noted. *, values are weighted mean (95\% confidence interval) or $\mathrm{n} / \mathrm{N}(\%) . \mathrm{BAV}$, balloon aortic valvuloplasty; Direct, direct TAVR without previous balloon aortic valvuloplasty; NR, not reported; PM, pacemaker; PPI, permanent pacemaker implantation; RBBB, right bundle branch block; TAVR, transcatheter aortic valve replacement.

implantation in comparison with previous observational studies (Figure 1).

\section{Evolut PRO vs. Acurate Neo}

Only one study met the inclusion criteria, the NEOPRO (A Multicenter Comparison of Acurate NEO Versus Evolut PRO Transcatheter Heart Valves) registry. After propensityscore matching, a total of 251 pairs were compared, without significant differences in baseline characteristics between Evolut PRO and Acurate Neo groups. The incidence of new PPI post-procedure was similar between groups (11\% Acurate neo vs. 12.8\% Evolut PRO, $\mathrm{P}=0.565)$. Results are displayed in Table 4.

Portico vs. Sapien 3 and Portico vs. commercially available prosthesis

Portico and Sapien 3 were compared in a propensitymatched study including 177 patients (Table 4). No statistically significant differences were observed between groups in terms of new PPI (21.9\% Portico vs. $17.5 \%$ Sapien 3, P>0.05). On the other hand, a randomized trial involving 732 TAVR recipients demonstrated higher rates of new PPI after Portico implantation in comparison with a pool of commercially available, mainly balloon-expandable, 
Table 2 Propensity-matched studies comparing the incidence of new-onset conduction disturbances between Sapien 3 and Evolut R/PRO valves

\begin{tabular}{|c|c|c|c|c|c|c|c|c|c|}
\hline Study & Cohorts & $\begin{array}{l}\text { Patients } \\
\text { (N) }\end{array}$ & Age & STS & Previous PM & $\begin{array}{l}\text { Previous } \\
\text { RBBB }\end{array}$ & $\begin{array}{l}\text { Post-TAVR } \\
\text { PPI }\end{array}$ & NOLBBB & Type of study \\
\hline \multirow[t]{2}{*}{$\begin{array}{l}\text { Enríquez-Rodríguez } \\
\text { et al. (15) }\end{array}$} & Sapien 3 & 80 & $82 \pm 6$ & $6.2 \pm 5$ & NR & NR & $6(7.5)$ & NR & $\begin{array}{l}\text { Propensity- } \\
\text { matched }\end{array}$ \\
\hline & Evolut $\mathrm{R}$ & 64 & $84 \pm 5$ & $5.8 \pm 5$ & NR & NR & $12(19.0)$ & NR & \\
\hline \multirow[t]{2}{*}{$\begin{array}{l}\text { Finkelstein } \\
\text { et al. (16) }\end{array}$} & Sapien 3 & 126 & 82 & 3.2 & $18(14.3)$ & NR & $-(12.8)$ & $31(24.8)$ & $\begin{array}{l}\text { Propensity- } \\
\text { matched }\end{array}$ \\
\hline & Evolut R & 126 & 82 & 3.2 & 16 (12.8) & NR & $-(12.8)$ & $34(26.7)$ & \\
\hline \multirow[t]{2}{*}{$\begin{array}{l}\text { Vlastra } \\
\text { et al. (17) }\end{array}$} & Sapien 3 & 1,122 & $81.5 \pm 7.1$ & 6.3 & NR & NR & $89(8.9)$ & NR & $\begin{array}{l}\text { Propensity- } \\
\text { matched }\end{array}$ \\
\hline & Evolut R & 1,091 & $81.3 \pm 7.1$ & 6.6 & NR & NR & $186(18.1)$ & NR & \\
\hline \multirow[t]{2}{*}{ Overall* } & Sapien 3 & 11,787 & $\begin{array}{l}82.8 \\
(82.8-82.9)\end{array}$ & $\begin{array}{l}6 \\
(5.95-6.05)\end{array}$ & $\begin{array}{l}2,119 / 10,585 \\
(20.0)\end{array}$ & - & [19.22] & $\begin{array}{l}31 / 126 \\
(24.8)\end{array}$ & - \\
\hline & Evolut R & 11,740 & $\begin{array}{l}82.9 \\
(82.9-90)\end{array}$ & $\begin{array}{l}6.22 \\
(6.17-6.28)\end{array}$ & $\begin{array}{l}2,107 / 10,585 \\
(19.9)\end{array}$ & - & [25.01] & $\begin{array}{l}34 / 126 \\
(26.7)\end{array}$ & - \\
\hline
\end{tabular}

Values are mean $\pm \mathrm{SD}$ or $\mathrm{n}(\%)$ except as noted. *, values are weighted mean (95\% confidence interval) or $\mathrm{n} / \mathrm{N}$ (\%). Values within [ ] represent the weighted mean of post-TAVR new PPI incidence across studies. NOLBBB, new onset left bundle branch block; NR, not reported; PM, pacemaker; PPI, permanent pacemaker implantation; RBBB, right bundle branch block; TAVR, transcatheter aortic valve replacement.

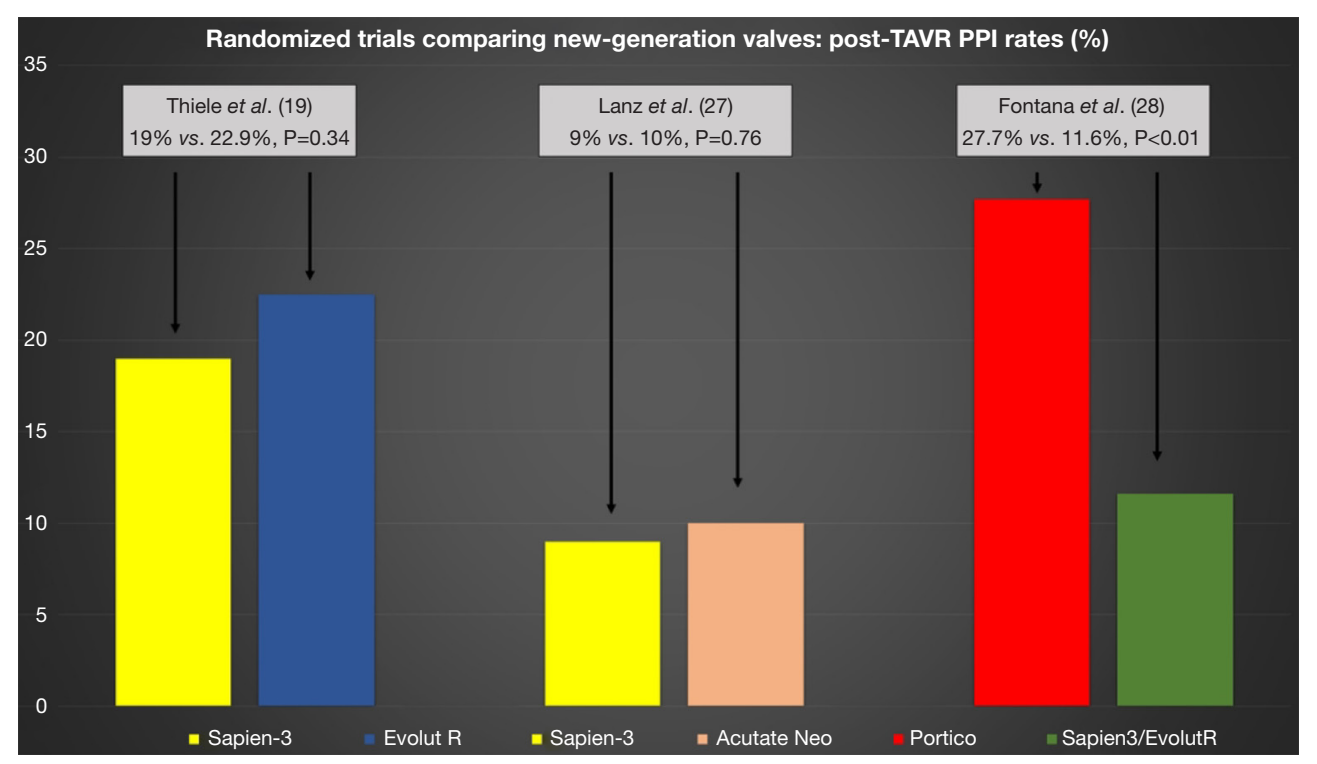

Figure 1 Pacemaker rates on randomized trials with direct comparison between new-generation TAVR devices. TAVR, transcatheter aortic valve replacement. 


\begin{tabular}{|c|c|c|c|c|c|c|c|c|c|c|}
\hline \multirow{2}{*}{ Variables } & \multicolumn{2}{|c|}{ Husser et al. (20) } & \multicolumn{2}{|c|}{ Husser et al. (21) } & \multicolumn{2}{|c|}{ Mauri et al. (22) } & \multicolumn{2}{|c|}{ Schaefer et al. (23) } & \multicolumn{2}{|l|}{ Overall* } \\
\hline & Sapien 3 & Acurate & Sapien 3 & Acurate & Sapien 3 & Acurate & Sapien 3 & Acurate & Sapien 3 & Acurate \\
\hline Patients (N) & 622 & 311 & 65 & 65 & 92 & 92 & 104 & 104 & 883 & 572 \\
\hline Age & $81 \pm 6$ & $81 \pm 6$ & $82[77-86]^{\#}$ & $81[77-84]^{\#}$ & $81.9 \pm 5.3$ & $82.8 \pm 6.5$ & $81.2 \pm 6.2$ & $81.7 \pm 5.5$ & $81.2(81.1-81.2)$ & $81.4(81.4-81.5)$ \\
\hline STS & NR & NR & NR & NR & NR & NR & $5.4 \pm 3.6$ & $5.8 \pm 3.8$ & 5.4 & 5.8 \\
\hline Previous PM & $62(10)$ & $28(9)$ & 0 & 0 & NR & NR & NR & NR & 62/687 (9) & 28/376 (7.4) \\
\hline $\begin{array}{l}\text { Previous } \\
\text { RBBB }\end{array}$ & $51(8.2)$ & $26(8.4)$ & 65 [100] & 65 [100] & NR & NR & NR & NR & 116/687 (16.9) & $91 / 376(24.2)$ \\
\hline \multirow[t]{2}{*}{$\begin{array}{l}\text { Post-TAVR } \\
\text { PPI }\end{array}$} & $87(15.5)$ & $28(9.9)$ & $29(44.6)$ & $15(23.1)$ & $14(15.2)$ & $11(12)$ & $17(16.4)$ & $11(10.6)$ & $\begin{array}{l}\text { a) } 147 / 821(17.9) \\
{[17.7]}\end{array}$ & $\begin{array}{l}\text { a) } 65 / 544(11.9) \\
{[11.9]}\end{array}$ \\
\hline & & & & & & & & & $\begin{array}{l}\text { b) } 118 / 756(15.6) \\
{[15.6]}\end{array}$ & $\begin{array}{l}\text { b) } 50 / 479(10.4) \\
{[10.4]}\end{array}$ \\
\hline NOLBBB & NR & NR & NR & NR & NR & NR & NR & NR & & \\
\hline
\end{tabular}

Values are mean $\pm \mathrm{SD}$ or $\mathrm{n}(\%)$ except as noted. *, values are weighted mean (95\% confidence interval) or $\mathrm{n} / \mathrm{N}(\%) .{ }^{*}$, values are median (interquartile range); a), post-TAVR new PPI rates considering all studies; b) post-TAVR new PPI rates excluding Husser et al. [2], as they were selected according to pre-procedure RBBB. Values are median (interquartile range). Values within [ ] represent the weighted mean of post-TAVR new PPI incidence across studies. NOLBBB, new onset left bundle branch block; NR, not reported; PM, pacemaker; PPI, permanent pacemaker implantation; RBBB, right bundle branch block; TAVR, transcatheter aortic valve replacement.

Table 4 Propensity-matched studies comparing Evolut R/PRO vs. Acurate Neo and Portico vs. Sapien 3 providing conduction disturbance rates

\begin{tabular}{|c|c|c|c|c|}
\hline Variables & \multicolumn{2}{|c|}{ Evolut R/PRO vs. Acurate Neo Pagnesi et al. (25) } & \multicolumn{2}{|c|}{ Portico vs. Sapien 3 Mas-Peiro et al. (26) } \\
\hline Patients (N) & 251 & 251 & 104 & 73 \\
\hline Age & $81.6 \pm 6.1$ & $81.4 \pm 6.5$ & $81.8 \pm 4.9$ & $81.5 \pm 7.3$ \\
\hline STS & $5.25 \pm 3.7$ & $5.08 \pm 3.05$ & $3.9 \pm 2.2$ & $3.9 \pm 2.9$ \\
\hline Previous RBBB & NR & NR & NR & NR \\
\hline Post-TAVR PPI & $29(12.8)$ & $25(11.0)$ & $20(21.9)$ & $11(17.5)$ \\
\hline NOLBBB & NR & NR & NR & NR \\
\hline
\end{tabular}

Values are mean \pm SD or $n(\%)$. NOLBBB, new onset left bundle branch block; NR, not reported; PM, pacemaker; PPI, permanent pacemaker implantation; RBBB, right bundle branch block; TAVR, transcatheter aortic valve replacement.

valves (27.7\% Portico vs. $11.6 \%$ commercially available) (Figure 1). The main characteristics and results from randomized trials comparing new-generation valves are displayed in Table 5.

\section{Implantation depth}

PubMed and Embase searches identified 958 and 2,284 records, respectively. After title and abstract revision as well as, duplicity elimination, 17 studies fulfilled the inclusion criteria and were selected. Overall, 1,784 Sapien 3, 421 Evolut R/PRO, 1,070 Acurate Neo and 298 Portico recipients were evaluated (Tables 6,7). The aortic angiography imaging immediately after valve deployment was the main technique used to measure ID (stent depth bellow the annulus in $\mathrm{mm}$ ). 


\begin{tabular}{|c|c|c|c|c|c|c|}
\hline \multirow[b]{2}{*}{ Variables } & \multicolumn{2}{|l|}{ Thiele et al. (19) } & \multicolumn{2}{|c|}{ Lanz et al. (27) } & \multicolumn{2}{|c|}{ Fontana et al. (28) } \\
\hline & Sapien 3 & Evolut R & Sapien 3 & Acurate Neo & Portico & $\begin{array}{l}\text { Commercially available; (65\% BEP; } 35 \% \\
\text { SEP\%); Sapien: } 4 \text { (1\%); Sapien XT: } 25(7 \%) \text {; } \\
\text { Sapien 3: } 206 \text { (57\%); CoreValve: } 15(4 \%) \text {; } \\
\text { Evolut R: } 90 \text { (25\%); Evolut PRO: } 22(6 \%)\end{array}$ \\
\hline Patients (N) & 219 & 219 & 367 & 372 & 371 & 361 \\
\hline Age & $81.5 \pm 5.7$ & $81.7 \pm 5.3$ & $83 \pm 3.9$ & $82.6 \pm 4.3$ & $83 \pm 7.6$ & $83.7 \pm 7$ \\
\hline STS & $7.6 \pm 7.4$ & $7.7 \pm 7.2$ & 3.4 & 3.7 & 6.4 & 6.6 \\
\hline Previous PM & 23 (10.5) & $24(11.0)$ & $36(9.8)$ & 43 (11.6) & $57(15.4)$ & 63 (17.5) \\
\hline Previous RBBB & NR & NR & NR & NR & NR & NR \\
\hline Post-TAVR PPI & 41/214 (19.2) & 49/213 (23.0) & $34 / 364(9.3)$ & $37 / 368$ (10.0) & $87 / 314$ (27.7) & $35 / 298$ (11.7) \\
\hline NOLBBB & NR & NR & NR & NR & NR & NR \\
\hline
\end{tabular}

Values are mean \pm SD or $n(\%)$. BEP, balloon-expandable prosthesis; NOLBBB, new onset left bundle branch block; NR, not reported; PM, pacemaker; PPI, permanent pacemaker implantation; RBBB, right bundle branch block; SEP, self-expandable prosthesis; TAVR, transcatheter aortic valve replacement.

The Sapien 3 was the valve with the largest body of evidence regarding ID. Two studies reported the lack of significant association between ID and pacemaker requirement $(30,35)$, and six studies showed an association between ID and either new PPI or a combination of PPI and any new conduction disturbance. Of note, 2 studies found an interaction between the ID and membranous septum (MS) length: one study showed a significant association between any conduction disturbance and two anatomical measures (MS length and the difference between the MS and ID) (41), and another study reported a directly proportional association between new PPI and ID (42).

Few studies evaluated the association between Evolut R/ PRO ID and conduction disturbances. One study involving 100 patients showed a significant correlation between ID and post-TAVR PPI rates (29). Recently, results on ID and MS length in 248 Evolut R/PRO patients were reported. The overall incidence of PPI was $9.7 \%$. The PPI rate increased as MS length decreased, and only the use of a larger Evolut valve size $(34 \mathrm{~mm})$ and a negative difference between ID and MS (deeper valve implantation than the length of the MS) were independently associated with PPI (OR 8.04; 95\% CI: 2.58-25.04). A valve implantation technique taking into consideration the MS length (targeting a valve ID < MS length) was prospectively evaluated in 100 patients, resulting in a much lower valve ID, which translated into a significant decrease in PPI rate, from $9.7 \%$ to $3 \%$ (44).
On the other hand, none of the four Acurate Neo studies evaluating the predictors of conduction disturbances showed an association prosthesis ID and PPI. Lastly, studies involving the Portico valve have revealed heterogeneous findings (one study showed a higher PPI rate in patients with deeper valve implantation, and other studies reported a lack of association between PPI and ID) (Table 6).

\section{Discussion}

The main findings from our systematic review on modifiable factors influencing the occurrence of conduction disturbances after TAVR can be summarized as follows: First, BAV does not seem to play any relevant role on postprocedural new PPI rate with new-generation devices. Second, a consistently slightly higher rate of PPI was observed in Evolut R/PRO recipients in comparison with balloon-expandable Sapien 3 ones. Third, Acurate Neo and Sapien 3 valves have been the new-generation prosthesis associated with the lowest PPI incidence, with controversial results between observational and randomized studies. Fourth, despite showing less consistency within Acurate Neo and Portico studies, ID and its relation with the MS length are anatomical and procedural landmarks that play a significant role in post-TAVR conduction disturbance rates. Fifth, considerable absence of data remains regarding NOLBBB among the main TAVR studies. 
Table 6 New-generation device studies with $\mathrm{n}>100$ evaluating the association between implantation depth and new-onset conduction abnormalities post-TAVR

\begin{tabular}{|c|c|c|c|c|c|c|}
\hline Study & $\begin{array}{l}\text { Number } \\
\text { of patients }\end{array}$ & $\begin{array}{l}\text { Valve ( } \mathrm{n} \text { if more } \\
\text { than one) }\end{array}$ & $\begin{array}{l}\text { ID assessment } \\
\text { technique }\end{array}$ & $\begin{array}{l}\text { Endpoint } \\
\text { evaluated }\end{array}$ & $\begin{array}{l}\text { Comparison } \\
\text { performed }\end{array}$ & Risk or difference \\
\hline $\begin{array}{l}\text { Gomes et al. } \\
\text { (29) }\end{array}$ & 200 & $\begin{array}{l}\text { CoreValve: } \\
\text { 100; Evolut R: } \\
100\end{array}$ & NR & PPI & $\begin{array}{l}\text { Multivariate regression } \\
\text { analysis: PPI as } \\
\text { dependent variable }\end{array}$ & $\begin{array}{l}\text { OR: } 1.2(P<0.001) \text { for } \\
\text { every } \mathrm{mm} \text { of ID }\end{array}$ \\
\hline $\begin{array}{l}\text { Sawaya et al. } \\
\text { (30) }\end{array}$ & 790 & $\begin{array}{l}\text { Sapien XT: } 507 \\
\text { Sapien 3: } 283\end{array}$ & $\begin{array}{l}\text { Angiographically: } \\
\mathrm{mm} \text { below septal side of } \\
\text { the frame }\end{array}$ & PPI & $\begin{array}{l}\text { Post-TAVR PPI vs. } \\
\text { non-PPI groups }\end{array}$ & $\begin{array}{l}5.3 \pm 2.4 \text { vs. } \\
5.0 \pm 2.6 \mathrm{~mm} ; \mathrm{P}=0.67\end{array}$ \\
\hline $\begin{array}{l}\text { De Torres-Alba } \\
\text { et al. (31) }\end{array}$ & 162 & Sapien 3 & $\begin{array}{l}\text { Angiographically } \\
\text { [aortic/ventricular stent } \\
\text { extension (\%)] }\end{array}$ & PPI & $\begin{array}{l}\text { Post-TAVR PPI vs. } \\
\text { non-PPI groups }\end{array}$ & $\begin{array}{l}72 / 28 \text { vs. } 67 / 23 ; \\
\mathrm{P}=0.032\end{array}$ \\
\hline $\begin{array}{l}\text { Husser et al. } \\
\text { (32) }\end{array}$ & 208 & Sapien 3 & $\begin{array}{l}\text { Angiographically } \\
\text { (\% of frame height below } \\
\text { the aortic annulus) }\end{array}$ & $\begin{array}{l}\mathrm{PPI} \text { and/or new } \\
\text { or worsened CA }\end{array}$ & $\begin{array}{l}\text { Post-TAVR PPI/CA vs. } \\
\text { non-PPI/CA }\end{array}$ & $\begin{array}{l}29 \% \pm 8 \% \text { vs. } 25 \% \pm 7 \% \\
P=0.003\end{array}$ \\
\hline $\begin{array}{l}\text { Schwerg et al. } \\
\text { (34) }\end{array}$ & 131 & Sapien 3 & $\begin{array}{l}\text { Angiographically: } \\
\text { mm between central } \\
\text { marker and aortic cusp }\end{array}$ & PPI & $\begin{array}{l}\text { High implantation } \\
\text { group ( }>2 \mathrm{~mm}) v \mathrm{~s} \text {. } \\
\text { low implantation } \\
\text { group }(<2 \mathrm{~mm})\end{array}$ & OR 9.7 (2.7-35.6) \\
\hline $\begin{array}{l}\text { Gonska et al. } \\
\text { (35) }\end{array}$ & 335 & Sapien 3 & $\begin{array}{l}\text { Angiographically: } \\
\text { mm below NCC }\end{array}$ & PPI & $\begin{array}{l}\text { Post-TAVR PPI vs. } \\
\text { non-PPI }\end{array}$ & $\begin{array}{l}6.7 \pm 2.59 \text { vs. } \\
6.39 \pm 2.48 \mathrm{~mm} ; P=0.43\end{array}$ \\
\hline $\begin{array}{l}\text { Mauri et al. } \\
\text { (24) }\end{array}$ & 212 & Acurate $\mathrm{Neo}$ & $\begin{array}{l}\text { Angiographically: } \\
\text { mm below NCC }\end{array}$ & PPI & $\begin{array}{l}\text { Post-TAVR PPI vs. } \\
\text { non-PPI }\end{array}$ & $\begin{array}{l}5.3 \pm 1.9 \text { vs. } \\
5.8 \pm 2.0 \mathrm{~mm} ; P=0.24\end{array}$ \\
\hline $\begin{array}{l}\text { Mas-Peiro } \\
\text { et al. (39) }\end{array}$ & 100 & Portico & Angiographically & PPI & $\begin{array}{l}\% \text { of PPI patients over } \\
\text { and below mean ID }\end{array}$ & $\begin{array}{l}81.3 \% \text { vs. } 18.7 \% \\
P=N R\end{array}$ \\
\hline $\begin{array}{l}\text { Walther et al. } \\
\text { (40) }\end{array}$ & 198 & Portico & $\begin{array}{l}\text { Angiographically: } \\
\text { mm below NCC }\end{array}$ & PPI & $\begin{array}{l}\text { Post-TAVR PPI vs. } \\
\text { non-PPI }\end{array}$ & $\begin{array}{l}6.35 \pm 2.11 \text { vs. } \\
6.07 \pm 2.28 ; P=0.50\end{array}$ \\
\hline
\end{tabular}

CA, conduction abnormality; ID, implantation depth; LBBB, left bundle branch block; NCC, non-coronary cusp; NR, not reported; OR, odds ratio; PPI, permanent pacemaker implantation; RBBB, right bundle branch block; TAVR, transcatheter aortic valve replacement. 


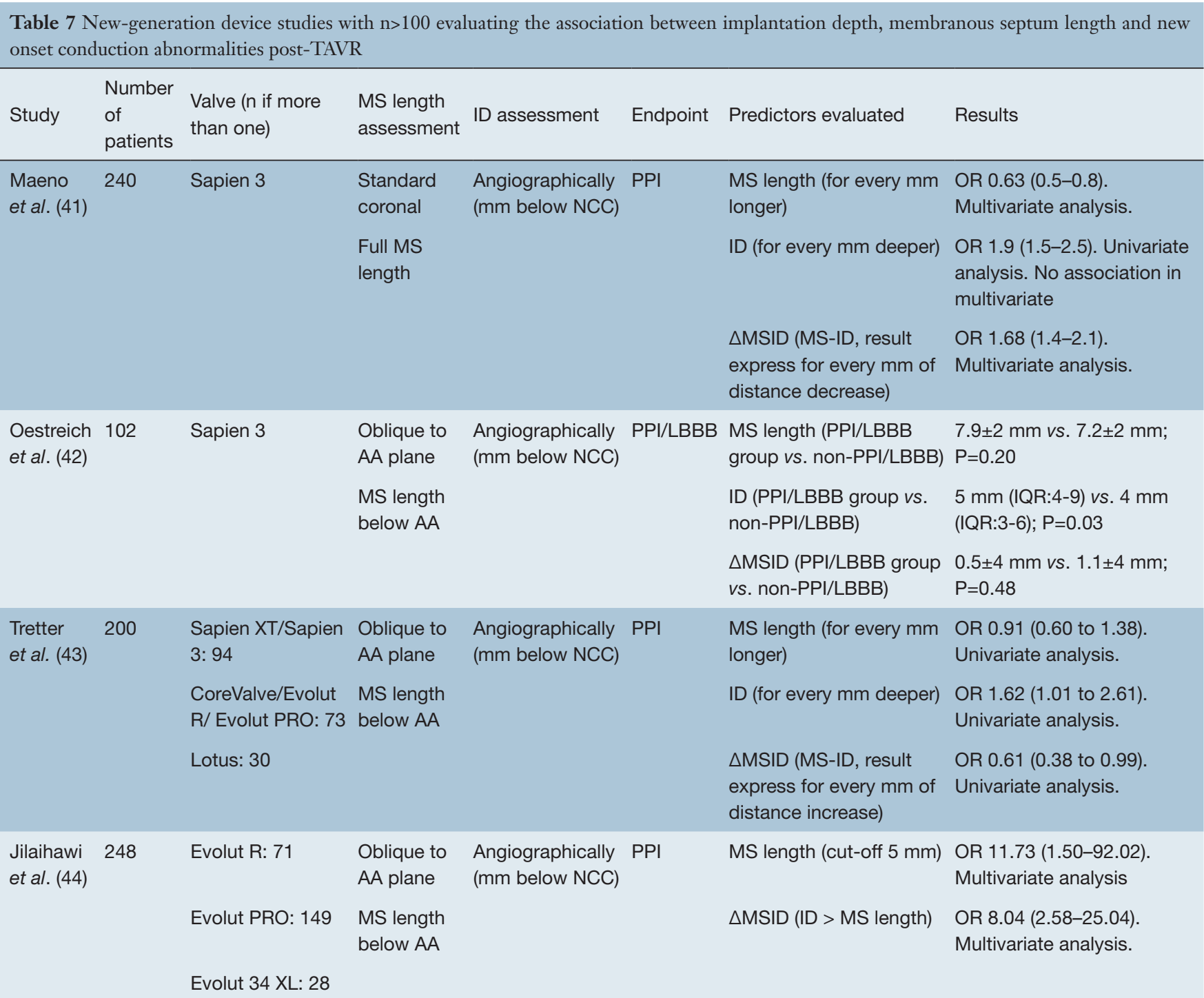

$\triangle M S I D$, difference between membranous septum length and implantation depth; AA, aortic annulus; ID, implantation depth; LBBB, left bundle branch block; MS, membranous septum; NCC, non-coronary cusp; OR, odds ratio; PPI, permanent pacemaker implantation; RBBB, right bundle branch block; TAVR, transcatheter aortic valve replacement.

\section{Balloon aortic valvuloplasty}

The most important studies evaluating BAV with newgeneration prosthesis (propensity-matched with more than 700 patients per group and a randomized trial) supported the lack of association between BAV and PPI post-TAVR. Dumonteil et al. (13) reported a higher PPI rate with the direct TAVR approach, with no clear explanation for that finding. It is out of the aim of this study to provide general conclusions on BAV apart from conduction disturbances. Therefore, considering the insight provided by this systematic review, BAV utilization should rely on other factors beyond PPI and/or conduction disturbance risk.

\section{Valve type comparison}

In light of the results of the present meta-analysis, the Acurate Neo and Sapien 3 seemed to be the new-generation valves associated with the lowest incidence of post-TAVR conduction disturbances, whereas the Evolut R/PRO valve exhibited slightly higher PPI rates. However, it should be highlighted 


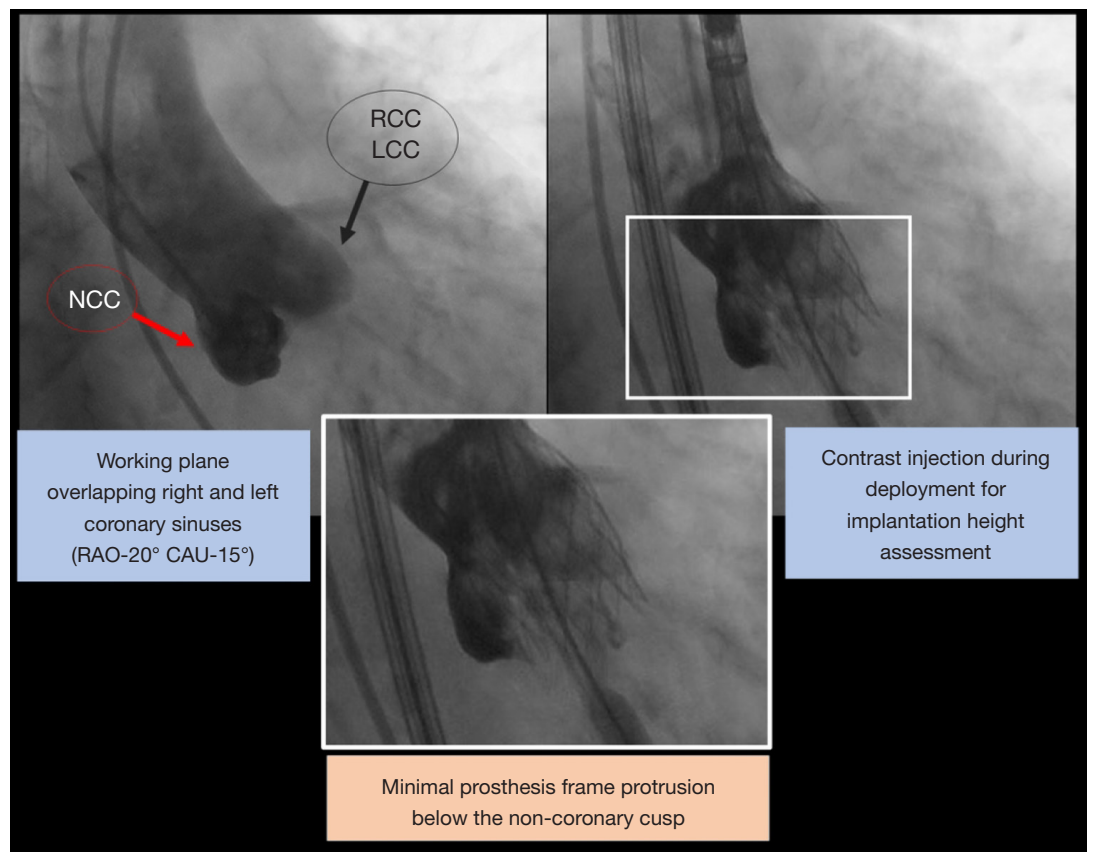

Figure $2 \mathrm{New}$ angiography working plane intended for limiting valve implantation depth. Superior left: aortography demonstrating right and left coronary sinuses overlapping (RCC and LCC black-encircled) opposite to the non-coronary sinus (NCC red-encircled). Superior right: Evolut R $29 \mathrm{~mm}$ deployment in the same working plane. Inferior: magnification demonstrating null frame protrusion below the noncoronary cusp.

that the gap between the Sapien and Evolut valves regarding PPI rates has notably decreased since the early-TAVR experience $(8,45)$, with a current difference of around $5 \%$ in PPI rate favoring the balloon-expandable valve system. On direct comparison between Acurate Neo and Sapien 3 valves, a trend towards a higher PPI rate with Sapien 3 was observed in propensity-matched studies, but a randomized trial showed a similar PPI rate between valve types (9\% Sapien 3 and $10 \%$ Acurate Neo). Thus, controversy remains regarding the risk of conduction disturbances between these valves, with current data not allowing for a clear position in favor of any of them. Acurate Neo and Evolut were compared on a propensity-matched cohort without significant differences, and results from the randomized SCOPE II (Safety and Efficacy Comparison of Two TAVI Systems in a Prospective Randomized Evaluation II) trial are eagerly awaited to shed more light on this topic. On the other hand, the Portico valve was associated with higher PPI rates in comparative studies with other new generation valves.

\section{Implantation depth}

Although less consistent in studies involving Acurate Neo and Portico patients, ID represents a modifiable factor impacting the occurrence of conduction disturbances postTAVR. It should be underscored that there was a substantial variability among studies on ID technique assessment and the methods linking ID and conduction disturbances. This is probably due to the lack of any specific consensus regarding ID assessment and the absence of an established cut-off point determining an increased risk of conduction disturbances. MS length has also been assessed by a variety of approaches. In our opinion, MS length below the aortic annular plane may be the most reliable landmark, since it considers the potential degree of interaction between the prosthesis frame and conduction tissue, which may be reduced by an "as-high-as-possible" implant. Interestingly, a new approach has been recently adopted in many centers in order to achieve a higher valve implant. Briefly, this approach considers an innovative angiography working plane during valve deployment in which both the right and left coronary sinuses are overlapped, thus isolating the non-coronary cusp (Figure 2). This might facilitate the deployment of the distal frame of the prosthesis just below or even at the same level as the aortic annular plane, which would translate into a decrease in ID. Future studies are 
warranted to validate the safety and efficacy of this new valve implantation technique.

\section{Limitations}

This study has the limitations inherent to a systematic review that collects only information described in the publications. Thus, relevant information may have been omitted. Moreover, this systematic review focused exclusively on modifiable factors which may play a role on the occurrence of conduction disturbances, and nonmodifiable factors (e.g., RBBB) were not included. Finally, other important topics like pre- and post-procedural ECG monitoring, PPI indications and management of conduction disturbances, which may be variable and add significant heterogeneity, were not considered (46).

\section{Acknowledgments}

Funding: JRC holds the Research Chair "Fondation Famille Jacques Larivière" for the Development of Structural Heart Disease Interventions.

\section{Footnote}

Conflicts of Interest: AA, GMC, DDV and LJ have been supported by grants from Martin Escudero foundation. JRC has received Institutional research grants from Edwards Lifesciences, Medtronic and Boston Scientific. LF has received grants from Institut Servier; and has received research grants from Biotronik, Edwards Lifesciences, and Medtronic. The other authors have no conflicts of interest to declare. The authors have no other relevant affiliations or financial involvement with any organization or entity with a financial interest in or financial conflict with the subject matter or materials discussed in the manuscript apart from those disclosed.

Open Access Statement: This is an Open Access article distributed in accordance with the Creative Commons Attribution-NonCommercial-NoDerivs 4.0 International License (CC BY-NC-ND 4.0), which permits the noncommercial replication and distribution of the article with the strict proviso that no changes or edits are made and the original work is properly cited (including links to both the formal publication through the relevant DOI and the license). See: https://creativecommons.org/licenses/by-nc-nd/4.0/.

\section{References}

1. Baumgartner H, Falk V, Bax JJ, et al. 2017 ESC/EACTS Guidelines for the management of valvular heart disease. Eur Heart J 2017;38:2739-91.

2. Nishimura RA, Otto CM, Bonow RO, et al. 2017 AHA/ ACC Focused Update of the 2014 AHA/ACC Guideline for the Management of Patients With Valvular Heart Disease: A Report of the American College of Cardiology/ American Heart Association Task Force on Clinical Practice Guidelines. Circulation 2017;135:e1159-95.

3. Reardon MJ, Van Mieghem NM, Popma JJ, et al. Surgical or Transcatheter Aortic-Valve Replacement in IntermediateRisk Patients. N Engl J Med 2017;376:1321-31.

4. Leon MB, Smith CR, Mack MJ, et al. Transcatheter or Surgical Aortic-Valve Replacement in Intermediate-Risk Patients. N Engl J Med 2016;374:1609-20.

5. Mack MJ, Leon MB, Thourani VH, et al. Transcatheter Aortic-Valve Replacement with a Balloon-Expandable Valve in Low-Risk Patients. N Engl J Med 2019;380:1695-705.

6. Popma JJ, Deeb GM, Yakubov SJ, et al. Transcatheter Aortic-Valve Replacement with a Self-Expanding Valve in Low-Risk Patients. N Engl J Med 2019;380:1706-15.

7. Faroux L, Chen S, Muntané-Carol G, et al. Clinical impact of conduction disturbances in transcatheter aortic valve replacement recipients: a systematic review and meta-analysis. Eur Heart J 2020;41:2771-81.

8. Auffret V, Puri R, Urena M, et al. Conduction disturbances after transcatheter aortic valve replacement: current status and future perspectives. Circulation 2017;136:1049-69.

9. Stewart LA, Clarke M, Rovers M, et al. Preferred reporting items for a systematic review and metaanalysis of individual participant data: The PRISMA-IPD statement. JAMA 2015;313:1657-65.

10. Abramowitz Y, Jilaihawi H, Chakravarty T, et al. Sapien 3 Transcatheter Aortic Valve Implantation with Moderate or Without Predilation. J Invasive Cardiol 2016;28:421-6.

11. Spaziano M, Sawaya F, Chevalier B, et al. Comparison of Systematic Predilation, Selective Predilation, and Direct Transcatheter Aortic Valve Implantation With the SAPIEN S3 Valve. Can J Cardiol 2017;33:260-8.

12. Giordano A, Corcione N, Ferraro P, Bedogni F, Testa $\mathrm{L}$ et al. Outcome of Patients Undergoing Transcatheter Aortic Valve Implantation After Prior Balloon Aortic Valvuloplasty. J Invasive Cardiol 2018;30:380-5.

13. Dumonteil N, Terkelsen C, Frerker C, Collart F, Wöhrle $\mathrm{J}$, Butter C, et al. Outcomes of transcatheter aortic valve 
replacement without predilation of the aortic valve: Insights from 1544 patients included in the SOURCE 3 registry. Int J Cardiol 2019;296:32-37.

14. Toutouzas K, Benetos G, Voudris V, et al. Pre-Dilatation Versus No Pre-Dilatation for Implantation of a SelfExpanding Valve in All Comers Undergoing TAVR: The DIRECT Trial. JACC Cardiovasc Interv 2019;12:767-77.

15. Enríquez-Rodríguez E, Amat-Santos IJ, Jiménez-Quevedo $\mathrm{P}$, et al. Comparison of the Hemodynamic Performance of the Balloon-expandable SAPIEN 3 Versus Self-expandable Evolut R Transcatheter Valve: A Case-matched Study. Rev Esp Cardiol 2018;71:735-42.

16. Finkelstein A, Steinvil A, Rozenbaum Z, et al. Efficacy and safety of new-generation transcatheter aortic valves: insights from the Israeli transcatheter aortic valve replacement registry. Clin Res Cardiol 2019;108:430-7.

17. Vlastra W, Chandrasekhar J, Muñoz-Garcia AJ, et al. Comparison of balloon-expandable vs. self-expandable valves in patients undergoing transfemoral transcatheter aortic valve implantation: From the CENTERcollaboration. Eur Heart J 2019;40:456-65.

18. Deharo P, Bisson A, Herbert J, et al. Impact of Sapien 3 Balloon-Expandable Versus Evolut R Self-Expandable Transcatheter Aortic Valve Implantation in Patients With Aortic Stenosis: Data From a Nationwide Analysis. Circulation 2020;141:260-8.

19. Thiele H, Kurz T, Feistritzer HJ, et al. Comparison of newer generation self-expandable vs. balloon-expandable valves in transcatheter aortic valve implantation: the randomized SOLVE-TAVI trial. Eur Heart J 2020;41:1890-9.

20. Husser O, Kim WK, Pellegrini C, et al. Multicenter Comparison of Novel Self-Expanding Versus BalloonExpandable Transcatheter Heart Valves. JACC Cardiovasc Interv 2017;10:2078-87.

21. Husser O, Pellegrini C, Kim W, et al. Transcatheter Valve SELECTion in Patients With Right Bundle Branch Block and Impact on Pacemaker Implantations. JACC Cardiovasc Interv 2019;12:1781-93.

22. Mauri V, Kim WK, Abumayyaleh M, et al. Short-Term Outcome and Hemodynamic Performance of NextGeneration Self-Expanding Versus Balloon-Expandable Transcatheter Aortic Valves in Patients with Small Aortic Annulus: A Multicenter Propensity-Matched Comparison. Circ Cardiovasc Interv 2017;10:1-7.

23. Schaefer A, Linder M, Seiffert M, et al. Comparison of latest generation transfemoral self-expandable and balloonexpandable transcatheter heart valves. Interact Cardiovasc
Thorac Surg 2017;25:905-11.

24. Mauri V, Deuschl F, Frohn T, et al. Predictors of paravalvular regurgitation and permanent pacemaker implantation after TAVR with a next-generation selfexpanding device. Clin Res Cardiol 2018;107:688-97.

25. Pagnesi M, Kim WK, Conradi L, et al. Transcatheter Aortic Valve Replacement With Next-Generation SelfExpanding Devices: A Multicenter, Retrospective, Propensity-Matched Comparison of Evolut PRO Versus Acurate neo Transcatheter Heart Valves. JACC Cardiovasc Interv 2019;12:433-43.

26. Mas-Peiro S, Seppelt PC, Weiler H, et al. A Direct Comparison of Self-Expandable Portico Versus BalloonExpandable Sapien 3 Devices for Transcatheter Aortic Valve Replacement: A Case-Matched Cohort Study. J Invasive Cardiol 2019;31:E199-204.

27. Lanz J, Kim WK, Walther T, et al. Safety and efficacy of a self-expanding versus a balloon-expandable bioprosthesis for transcatheter aortic valve replacement in patients with symptomatic severe aortic stenosis: a randomised noninferiority trial. Lancet 2019;394:1619-28.

28. Fontana GP. A randomized trial of Portico vs. commercially available transcatheter aortic valves in patients with severe aortic stenosis. Presented at: TCT 2019. September 27, 2019. San Francisco, CA.

29. Gomes B, Geis NA, Chorianopoulos E, et al. Improvements of Procedural Results With a NewGeneration Self-Expanding Transfemoral Aortic Valve Prosthesis in Comparison to the Old-Generation Device. J Interv Cardiol 2017;30:72-8.

30. Sawaya FJ, Spaziano M, Lefèvre T, et al. Comparison between the SAPIEN S3 and the SAPIEN XT transcatheter heart valves: A single-center experience. World J Cardiol 2016;8:735.

31. De Torres-Alba F, Kaleschke G, Diller GP, et al. Changes in the Pacemaker Rate after Transition from Edwards SAPIEN XT to SAPIEN 3 Transcatheter Aortic Valve Implantation the Critical Role of Valve Implantation Height. JACC Cardiovasc Interv 2016;9:805-13.

32. Husser O, Pellegrini C, Kessler T, et al. Predictors of Permanent Pacemaker Implantations and New-Onset Conduction Abnormalities With the SAPIEN 3 BalloonExpandable Transcatheter Heart Valve. JACC Cardiovasc Interv 2016;9:244-54.

33. Mauri V, Reimann A, Stern D, et al. Predictors of Permanent Pacemaker Implantation After Transcatheter Aortic Valve Replacement With the SAPIEN 3. JACC Cardiovasc Interv 2016;9:2200-9. 
34. Schwerg M, Fulde F, Dreger H, et al. Optimized Implantation Height of the Edwards SAPIEN 3 Valve to Minimize Pacemaker Implantation After TAVI. J Interv Cardiol 2016;29:370-4.

35. Gonska B, Seeger J, Keßler M, et al. Predictors for permanent pacemaker implantation in patients undergoing transfemoral aortic valve implantation with the Edwards Sapien 3 valve. Clin Res Cardiol 2017;106:590-7.

36. Kim WK, Möllmann H, Liebetrau C, et al. The ACURATE neo Transcatheter Heart Valve: A Comprehensive Analysis of Predictors of Procedural Outcome. JACC Cardiovasc Interv 2018;11:1721-9.

37. Toggweiler S, Nissen H, Mogensen B, et al. Very low pacemaker rate following ACURATE neo transcatheter heart valve implantation. EuroIntervention 2017;13:1273-80.

38. Pellegrini C, Husser O, Kim WK, et al. Predictors of Need for Permanent Pacemaker Implantation and Conduction Abnormalities With a Novel Self-expanding Transcatheter Heart Valve. Rev Esp Cardiol 2019;72:145-53.

39. Mas-Peiro S, Vasa-Nicotera M, Weiler H, et al. ThirtyDay Outcomes in 100 Consecutive Patients Undergoing Transfemoral Aortic Valve Replacement With the Portico Valve on an All-Comer Basis. J Invasive Cardiol 2017;29:431-6.

40. Walther T, Manoharan G, Linke A, et al. Incidence of new-onset left bundle branch block and predictors of new permanent pacemaker following transcatheter aortic valve replacement with the Portico ${ }^{\mathrm{TM}}$ valve. Eur J Cardiothorac Surg 2018;54:467-74.

41. Maeno Y, Abramowitz Y, Kawamori H, et al. A Highly Predictive Risk Model for Pacemaker Implantation After TAVR. JACC Cardiovasc Imaging 2017;10:1139-47.

42. Oestreich BA, Mbai M, Gurevich S, et al. Computed tomography (CT) assessment of the membranous septal anatomy prior to transcatheter aortic valve replacement (TAVR) with the balloon-expandable SAPIEN 3 valve. Cardiovasc Revasc Med 2018;19:626-31.

43. Tretter JT, Mori S, Anderson RH, et al. Anatomical predictors of conduction damage after transcatheter implantation of the aortic valve. Open Heart 2019;6:e000972.

44. Jilaihawi H, Zhao Z, Du R, et al. Minimizing Permanent Pacemaker Following Repositionable Self-Expanding Transcatheter Aortic Valve Replacement. JACC Cardiovasc Interv 2019;12:1796-807.

45. Erkapic D, De Rosa S, Kelava A, et al. Risk for permanent pacemaker after transcatheter aortic valve implantation: A comprehensive analysis of the literature. J Cardiovasc Electrophysiol 2012;23:391-7.

46. Rodés-Cabau J, Ellenbogen KA, Krahn AD, et al. Management of conduction disturbances associated with transcatheter aortic valve replacement: JACC Scientific Expert Panel. J Am Coll Cardiol 2019;74:1086-106.
Cite this article as: Alperi A, Muntané-Carol G, Freitas-Ferraz AB, Junquera L, del Val D, Faroux L, Philippon F, RodésCabau J. Overcoming the transcatheter aortic valve replacement Achilles heel: conduction abnormalities-a systematic review. Ann Cardiothorac Surg 2020;9(6):429-441. doi: 10.21037/acs2020-av-40 